\title{
Visual Target Tracking and Relative Navigation for Unmanned Aerial Vehicles in a GPS-Denied Environment
}

\author{
Youngjoo Kim*, Wooyoung Jung** and Hyochoong Bang*** \\ Korea Advanced Institute of Science and Technology, Daejeon, South Korea
}

\begin{abstract}
We present a system for the real-time visual relative navigation of a fixed-wing unmanned aerial vehicle in a GPS-denied environment. An extended Kalman filter is used to construct a vision-aided navigation system by fusing the image processing results with barometer and inertial sensor measurements. Using a mean-shift object tracking algorithm, an onboard vision system provides pixel measurements to the navigation filter. The filter is slightly modified to deal with delayed measurements from the vision system. The image processing algorithm and the navigation filter are verified by flight tests. The results show that the proposed aerial system is able to maintain circling around a target without using GPS data.
\end{abstract}

Key words: unmanned aerial vehicle, target tracking, relative navigation, delayed measurement, GPS-denied, vision-aided navigation, extended Kalman filter

\section{Introduction}

Unmanned aerial vehicles (UAVs) have been exploited widely in military and civilian operations, particularly in extreme environment, such as reconnaissance and surveillance, disaster observation, and rescue missions. For any aerial vehicle, the ability to navigate is important to carry out the operations. One of the first techniques for UAV navigation was an inertial navigation system (INS). INS is used in most conventional aerial vehicle navigation [1]. However, INS suffers from error accumulation because it estimates current position and velocity by integrating acceleration data. That is, the magnitude of the estimation error with INS increases with time. Thus, global positioning system (GPS) is often used as a supplement to recalibrate the INS periodically, through an INS/GPS navigation system [2, 3].

Although GPS is a primary navigation method used for ground and air vehicles, several weak points of GPS associated with signal disruptions are also known. Signal disruptions include unintentional disruptions, such as ionosphere interference and RF interference, and intentional disruptions, involving spoofing, jamming, and meaconing. Researchers have been investigating strategies to relieve the weak point of the current GPS navigation technique. GPS backup methods include instrument landing systems (ILS) and long range navigation (LORAN) [4]. However, these modalities are limited by the range of their ground transmitters, so may not be feasible for UAVs operating in remote sites. Based on this restriction, local methods for estimating UAV position have been studied widely in recent years.

One alternative that will be considered in this paper is a vision-aided navigation system. While there are many different approaches for using camera images in navigation, this paper focuses on implementing a real-time navigation system that can operate with sudden and temporal GPS signal disruption. One of the most basic and rapid techniques in image processing is to track an object. Once a vision system detects and tracks a target, it can extract position information of the target or, in an opposite way, the relative position of the vision system with respect to the target. Based on this, a vision system would be expected to be able to compensate for INS integration error when a target is visible. A similar approach has been proposed [5] but it provides simulation results from aerial images. Moreover, the image-processing algorithm for
This is an Open Access article distributed under the terms of the Creative Commons Attribution Non-Commercial License (http://creativecommons.org/licenses/by$\mathrm{nc} / 3.0 /$ which permits unrestricted non-commercial use, distribution, and reproduction in any medium, provided the original work is properly cited. (c) $\quad$ Ph. D Student, Corresponding author: yjkim@ascl.kaist.ac.kr
** Ph. D Student $* * *$ Professor 
the system requires relatively high computational power. On the other hand, this paper proposes practical algorithms and assesses their feasibility with flight tests.

In this paper, we propose an INS/vision navigation system that can deal with a GPS-denied environment. At the moment of GPS blackout, the vision system of the UAV is expected to track any landmark or ground feature for recalibrating INS data and perform a position-keeping maneuver. A kernelbased mean-shift algorithm is used for visual target tracking. Because the height information is crucial to the vision-aided navigation, height measurements from GPS are used as if a barometer is on the UAV. Once detected, the target is tracked in successive images by the algorithm with fast computation. The target tracking algorithm runs on an image-processing board on the UAV. The proposed navigation system is based on an extended Kalman filter (EKF) to estimate the UAV's position from image processing results and sensory acceleration data from an inertial measurement unit (IMU). Typically, the image processing results are time-delayed because the results are not from a current image. To obtain the correct solution from the filter, we provide a method to deal with the delayed measurement. Finally, a flight test was conducted using a fixed-wing UAV with a two-axis gimbal and onboard vision system.

The following sections are organized as follows. Section 2 describes the image processing algorithms to track the target. In Section 3, an EKF-based navigation filter design is addressed. The flight test is conducted with flight test set-up presented in Section 4 and the results are shown in Section 5. In Section 6, we provide a summary and our conclusions.

\section{Image-Processing Algorithms}

In this section, a kernel-based algorithm [6], also known as "mean-shift," is used for real-time object tracking. The algorithm has been used in not only object tracking, but also other vision tasks, such as image clustering and segmentation.

The mean-shift algorithm uses kernel data and histograms of pixel values of the image. In every video sequence, the tracking algorithm calculates similarity with object and candidate data to compute the mean-shift vector. The object position in the current image sequence can be estimated with past object positions, mean-shift vectors, and a hypothesis: the object moved as a mean-shift vector. In the mean-shift algorithm, the object and candidate are represented with feature space as

$$
\hat{q}=\left\{\hat{q}_{u}\right\}_{u=1 \ldots m}, \quad \sum_{u=1}^{m} \hat{q}_{u}=1
$$

$$
\hat{p}(y)=\left\{\hat{p}_{u}(y)\right\}_{u=1 \ldots m}, \quad \sum_{u=1}^{m} \hat{p}_{u}=1
$$

where $\mathrm{m}$ is the bin of histogram. If the object model is located at $\left\{\boldsymbol{x}_{i}^{*}\right\}_{i=1 \ldots n}$ with $\mathrm{m}$-bins histogram, the feature space of the object can be written as

$$
\hat{q}_{u}=C_{q} \sum_{i=1}^{n} k\left(\left\|x_{i}^{*}\right\|^{2}\right) \delta\left[b\left(x_{i}^{*}\right)-u\right]
$$

where $\hat{q}_{u}$ is the feature space of the model, $\delta$ is a kronecker delta function, and $C_{q}$ is a normalization constant. Normalization constant can be calculated by

$$
C_{q}=\frac{1}{\sum_{i=1}^{n} k\left(\left\|x_{i}^{*}\right\|^{2}\right)}
$$

where $\mathrm{k}(x)$ is the kernel function. While there are many kernel functions, we used the Epanechnikov kernel function, as follows.

$$
k(x)=\left\{\begin{array}{cl}
\frac{1}{2} c_{d}(d+2)(1-x) & , \text { if } \mathrm{x}<1 \\
0 & , \text { otherwise }
\end{array}\right.
$$

where $c_{d}$ is the unit volume, $d$ is the channel, and $x$ is the pixel location.

In the same way, the feature space of a candidate and normalization constant are expressed as

$$
\begin{aligned}
& \hat{p}_{u}(y)=C_{h} \sum_{i=1}^{n h} k\left(\left\|\frac{y-x_{i}}{h}\right\|^{2}\right) \delta\left[b\left(x_{i}\right)-u\right] \\
& C_{h}=\frac{1}{\sum_{i=1}^{n h} k\left(\left\|\frac{y-x_{i}}{h}\right\|^{2}\right)}
\end{aligned}
$$

where $\mathrm{h}$ is the bandwidth. After computing the object and candidate feature space data, the weighting can be calculated as

$$
w_{i}=\sum_{u=1}^{m} \sqrt{\frac{\hat{q}_{u}}{\hat{p}_{u}\left(y_{0}\right)}} \delta\left[b\left(x_{i}\right)-u\right]
$$

where $y_{0}$ is the center position of the currently estimated object. With this weighting result, the mean-shift vector can be obtained as

$$
\hat{y}_{1}=\frac{\sum_{i=1}^{n h} x_{i} w_{i} g\left(\frac{\hat{y}_{0}-x_{i}}{h}\right)^{2}}{\sum_{i=1}^{n h} w_{i} g\left(\frac{\hat{y}_{0}-x_{i}}{h}\right)^{2}}
$$

where $y_{0}$ is the center position of past estimated object and $\mathrm{g}(\mathrm{x})$ is given by 


$$
g(x)=-k^{\prime}(x)
$$

Because $g(x)$ becomes constant in the Epanechnikov kernel function, we can rewrite the mean-shift vector as

$$
\hat{y}_{1}=\frac{\sum_{i=1}^{n h} x_{i} w_{i}}{\sum_{i=1}^{n h} w_{i}}
$$

Thus, the estimated object position is shifted as a meanshift vector. We iterate this whole procedure at every video sequence until the mean-shift vector meets a tolerance or the number of iterations exceeds a predetermined limit. A flow chart of whole procedure is shown in Fig. 1.

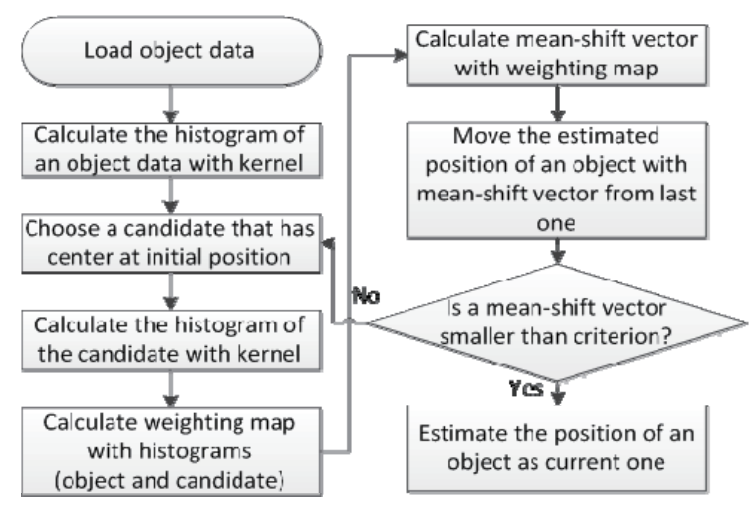

Fig. 1. Flow chart of the mean-shift tracking procedure.

The mean-shift algorithm does not search an object in every area in an image. It searches the object only in the region of interest, centered on the previous object location. Thus, the initial position or previous position should be given. In the first image, an additional algorithm for detecting object(s) is needed. In this study, we give the initial position by clicking a target on the image, using the mission control system (MCS). Fig. 2 shows the MCS and

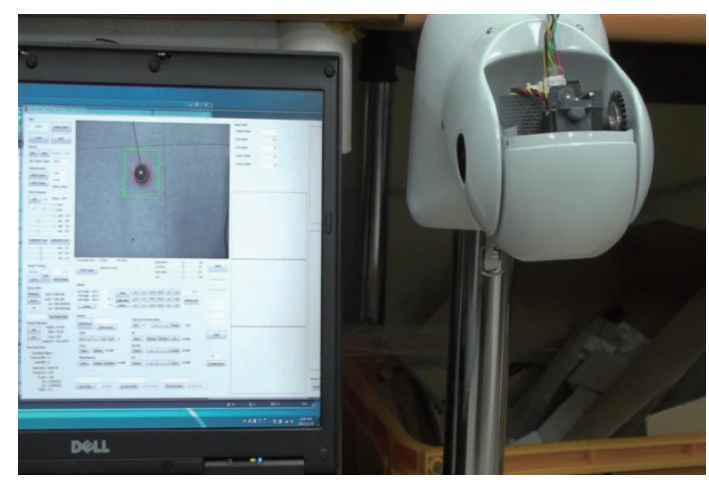

Fig. 2. Mission control system (MCS) and a two-axis gimbal system. If we click an object on the image, the vision system starts tracking the target. The gimbal system is autonomously controlled to locate the target at the center of the image. gimbal system. The airborne target tracking results are shown in Fig. 3.

\section{Relative Navigation in GPS-denied Envi- ronment}

In this section, we describe a real-time navigation filter designed to estimate the UAV position from the image processing outputs obtained in Section 2 and inertial sensor measurements. Fig. 4 depicts the system of vision-aided relative navigation. The navigation system switches to INS/ vision navigation when a GPS signal is denied. It estimates the UAV position relative to a target or a landmark the position of which is unknown. The estimated UAV state is transferred to the guidance and control block, which generates actuator inputs.

\subsection{Coordinate Frames}

The coordinate frames are defined to express state variables as follows. The local reference frame is a frame that is fixed locally to a target, as shown in Fig. 5. The
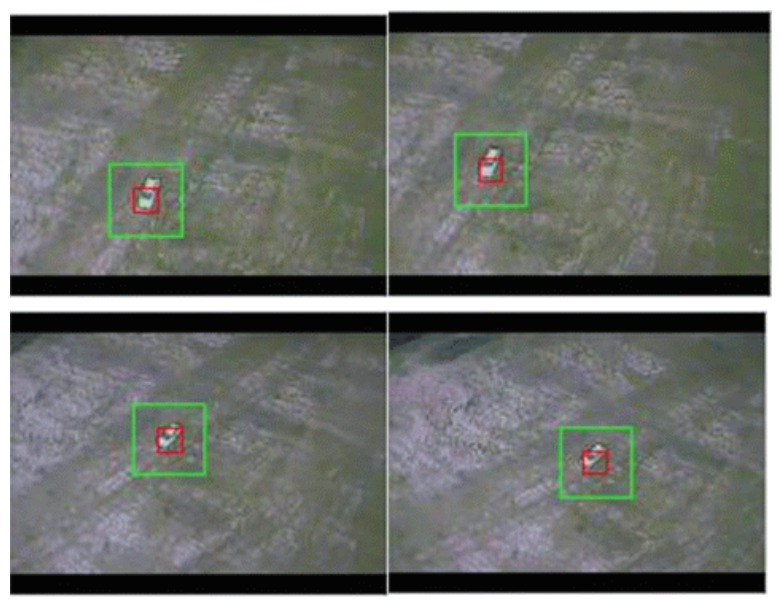

Fig. 3. Target tracking results in flight test. The outer blue box denotes region of interest $(\mathrm{ROI})$ and inner red box denotes the target.

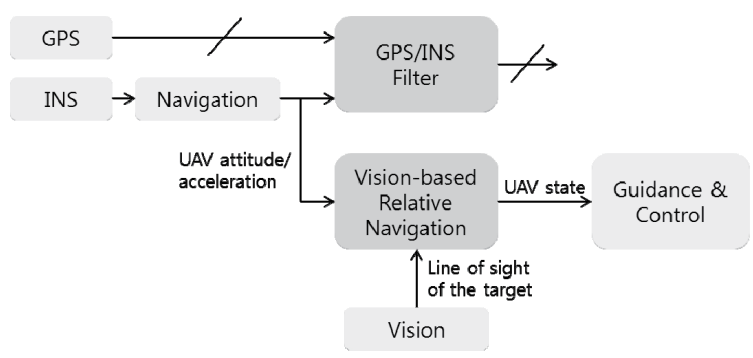

Fig. 4. Diagram of vision-aided relative navigation system, also denoted as INS/vision navigation. 
frame is newly defined when GPS is denied. Thus, the UAV position is expressed in East-North-Up (ENU) coordinates, the origin of which is at the center of the target. Neglecting rotation and curvature of the Earth, this frame is used as a temporal inertial frame, $F_{n}$, at the moment of GPS blackout for navigational purposes. If a GPS signals become available, the target position and UAV position are recovered to inertial coordinates.

The UAV body frame, $F_{b}$, is a frame fixed to the UAV body. The center of gravity of the body is the origin. The $\mathrm{X}$-axis is pointing forward and the Z-axis is pointing downwards. The vehicle frame, denoted as $F_{y}$, originates at the vehicle's center of mass with the $\mathrm{X}$-axis directed North, the Y-axis directed East, and the Z-axis directed towards the center of the earth. The attitude of the UAV is defined by Euler angles of the body frame with respect to vehicle frame. Additionally, coordinate frames for the camera image should be defined to convert pixel measurements to inertial positions. One is the gimbal frame, denoted as $F_{g}$. The gimbal frame originates at the gimbal rotation center. The gimbal is placed on the UAV body with known angles. The camera frame, $F_{c}$, originates at the optical center. The pixel coordinates of the camera frame can be transformed to inertial coordinates by multiplying coordinate transformation matrices, which will not be addressed in detail here. In this paper, a matrix for coordinate transformation from $F_{i}$ to $F_{j}$ is denoted as $T_{i}^{j}$. Refer to the paper [7], which describes coordinate frame transformations specific to ground target tracking. If relative position between coordinate frames is not considered, a rotation matrix $R_{i}^{j}$ will be used.

\subsection{Navigation Filter Design}

In this study, the inertial measurements and visual measurements are blended by EKF $[8,9]$. EKF can deal with nonlinear measurement model and it does not require high computational power. The process model and measurement

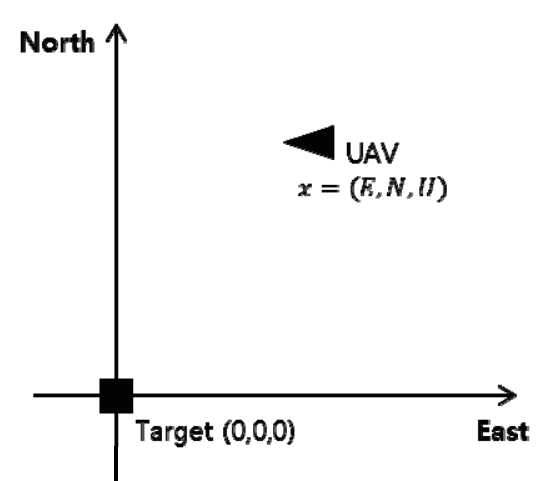

Fig. 5. Newly defined ENU frame where target position is the origin. model are designed as follows.

\subsubsection{Process model}

The estimator state is defined as

$$
x=\left[E, N, U, V_{E}, V_{N}, V_{U}\right]^{T}
$$

which includes UAV position and velocity in an inertial frame. The process model is represented as time derivatives of position and velocity.

$$
\dot{x}=\left[\begin{array}{c}
\dot{E} \\
\dot{N} \\
\dot{U} \\
\dot{V}_{E} \\
\dot{V}_{N} \\
\dot{V}_{U}
\end{array}\right]=\left[\begin{array}{c}
V_{E} \\
V_{N} \\
V_{U} \\
a_{E} \\
a_{N} \\
a_{U}
\end{array}\right]+\left[\begin{array}{c}
0 \\
0 \\
0 \\
\eta_{E} \\
\eta_{N} \\
\eta_{U}
\end{array}\right]=f(x, u)+G(x) \eta
$$

where the acceleration of UAV in inertial frame, $\left[a_{E}, a_{N}, a_{U}\right]^{T}$, is obtained from the outputs of the inertial measurement unit. The body acceleration is transformed to acceleration in the inertial frame as

$$
\left[\begin{array}{l}
a_{E} \\
a_{N} \\
a_{U}
\end{array}\right]=R_{v}^{n} R_{b}^{v}\left[\begin{array}{l}
a_{x} \\
a_{y} \\
a_{z}
\end{array}\right]
$$

where the rotation matrix $R_{b}{ }^{v}$ is calculated by attitude angle estimates of IMU. Note that the coordinate transform matrices are not included in the filter models so that we can reduce filter operation load. In this study, computational efficiency is regarded as very important to enable real-time application.

In Eq. (13), $\eta_{E}, \eta_{N}$, and $\eta_{U}$ denote the process noise. The process noise has a zero-mean, and its covariance matrix is given by

$$
\begin{gathered}
E\left[\eta(t) \eta(\tau)^{T}\right]=Q \delta(t-\tau) \\
Q=\left[\begin{array}{ccc}
\sigma_{\eta_{E}}^{2} & 0 & 0 \\
0 & \sigma_{\eta_{N}}^{2} & 0 \\
0 & 0 & \sigma_{\eta_{U}}^{2}
\end{array}\right]
\end{gathered}
$$

The values of the standard deviation of the process noise were determined by matching empirical data collected during flight tests.

The process model, Eq. (13), can be discretized as

$$
\begin{aligned}
& x_{k+1}=\Phi_{k} x_{k}+w_{k} \\
& \Phi_{k} \cong I+\left.\frac{\partial f}{\partial x}\right|_{x_{k}=\hat{x}_{k}} \Delta t
\end{aligned}
$$

where $w_{k}$ is the discretized zero-mean process noise. Its covariance matrix is approximated as follows.

$$
Q_{k} \cong G Q G^{T} \Delta t
$$




\subsubsection{Measurement Model}

The measurement vector is composed of line-of-sight angles of the target, azimuth and elevation, and altitude of the UAV. The line-of-sight is expressed by a set of two angles between the UAV and the target, defined in the inertial frame. The measurement model below expresses the non-linear relationship between UAV position, line-of-sight angles, and altitude.

$$
z_{k}=\left[\begin{array}{c}
A z \\
E l \\
h
\end{array}\right]+v_{k}=\left[\begin{array}{c}
\tan ^{-1} \frac{E}{N} \\
\tan ^{-1} \frac{U}{\sqrt{E^{2}+N^{2}}} \\
U+U_{0}
\end{array}\right]+\left[\begin{array}{c}
v_{A z} \\
v_{E l} \\
v_{h}
\end{array}\right]=h\left(x_{k}\right)+v_{k}
$$

In the measurement model above, $v_{k}=\left[v_{A z}, v_{E l}, v_{h}\right]^{T}$ represents zero-mean measurement noise whose covariance matrix is expressed as

$$
R_{k}=\left[\begin{array}{ccc}
\sigma_{A z}^{2} & 0 & 0 \\
0 & \sigma_{E l}^{2} & 0 \\
0 & 0 & \sigma_{h}^{2}
\end{array}\right]
$$

In a manner similar to the process noise model development, the values of the standard deviation of the measurement noise were determined by matching empirical data collected during flight tests.

The line-of-sight is measured by the image processing system. When the target is detected in the image, the pixel coordinates of the target are obtained in the camera frame as

$$
\vec{x}_{c}=\left[x_{c}, y_{c},-f\right]^{T}
$$

where $f$ is focal length of the camera. Thus, the vector pointing to the target from the UAV is calculated as

$$
\vec{x}_{n}=R_{v}^{n} R_{b}^{v} R_{g}^{b} R_{c}^{g} \vec{x}_{c}=[e, n, u]_{\text {target }}^{T}=-[e, n, u]_{\text {UAV }}^{T}
$$

In above equation, $[e, n, u]_{U A V}^{T}$ is a vector pointing to the UAV from the target. The line-of- sight angles are calculated with this vector and used as the measurement.

Note that $\vec{x}_{n}$ does not mean the relative position of the target with respect to the UAV. Because altitude information is given, it is possible to obtain the distance between the UAV and the target. This is the reason why an altitude measurement $h$ is needed. The altitude is measured by a barometer, and the reference height $U_{0}$ where barometer output denotes zero is assumed to be known.

\subsubsection{Extended Kalman Filter}

An extended Kalman filter is applied to the discrete system in Eq. (16). Let $\hat{x}_{k}^{-}$and $\hat{x}_{k}^{+}$denote the propagated and updated state estimates at time $t_{k}$, respectively. Then,
$P_{k}^{-}$and $P_{k}^{+}$are the corresponding error covariance matrices. The EKF is composed of the time propagation step and the measurement update step.

The estimator state and its error covariance matrix are propagated as follows.

$$
\begin{aligned}
& \hat{x}_{k}^{-}=\hat{x}_{k-1}^{+}+f\left(t, \hat{x}_{k-1}^{+}, u_{k-1}\right) \Delta t \\
& P_{k}^{-}=\Phi_{k-1} P_{k-1}^{+} \Phi_{k-1}^{T}+Q_{k-1}
\end{aligned}
$$

When the measurement $z_{k}$ is obtained, the estimator state is updated as follows.

$$
\begin{aligned}
& \hat{x}_{k}^{+}=\hat{x}_{k}^{-}+K_{k}\left(z_{k}-\hat{z}_{k}^{-}\right) \\
& P_{k}^{+}=\left(I-K_{k} H_{k}\right) P_{k}^{-}
\end{aligned}
$$

where

$$
\begin{aligned}
& \hat{z}_{k}^{-}=h\left(\hat{x}_{k}^{-}\right) \\
& H_{k}=\left.\frac{\partial h}{\partial x}\right|_{x_{k}=\hat{x}_{k}^{-}} \\
& K_{k}=P_{k}^{-} H_{k}^{T}\left(H_{k} P_{k}^{-} H_{k}^{T}+R_{k}\right)^{-1}
\end{aligned}
$$

Typically, the acceleration and attitude measurements from IMU are delivered at a higher frequency than the pixel measurements from the image processing system. In this study, the propagation step is conducted at $25 \mathrm{~Hz}$ and the update step is conducted at $5 \mathrm{~Hz}$.

\subsection{Delayed Measurements}

Before directly implementing the EKF for visionaided relative navigation, consideration of time-delayed measurements is addressed. In a real system, the pixel

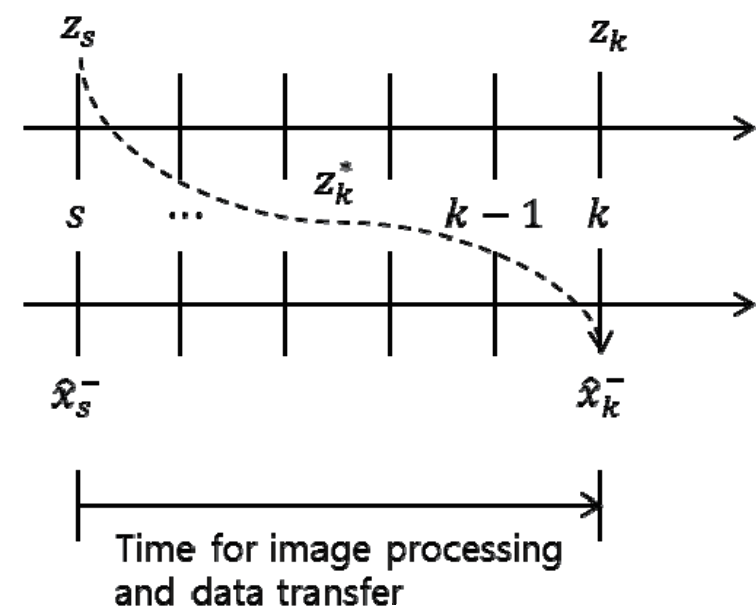

Fig. 6. System with a delayed measurement. 
measurement is obtained after image processing is completed. Thus, there is a delay in delivering the measurement vector from the image processing processor to the flight control computer. From the perspective of the navigation filter, the measurement at time $s$ arrives at time $k$ as shown in Fig. 6 . That is, the visual measurement arriving at time $k$ was extracted from the image captured at time $s$. It cannot be fused using the normal Kalman filter equations.

Thus, the filter requires some modifications in the structure [10]. The filter state at time $k$ without update of delayed measurement $z_{k}^{*}$ is expressed as

$$
\hat{x}_{k}^{-}=\left(\prod_{i=s}^{k-1} \Phi_{i}\right) \hat{x}_{s}^{-}
$$

When the delayed measurement arrives, it is used to update filter state $\hat{x}_{s}^{-}$and the resulting state at time $k^{-}$, denoted as $\hat{x}_{k}^{-1}$, can be derived as follows.

$$
\begin{aligned}
\hat{x}_{k}^{-\prime} & =\left(\prod_{i=s}^{k-1} \Phi_{i}\right) \hat{x}_{s}^{+} \\
& =\left(\prod_{i=s}^{k-1} \Phi_{i}\right)\left(\hat{x}_{s}^{-}+K_{s}\left(z_{k}^{*}-H_{s} \hat{x}_{s}^{-}\right)\right) \\
& =\left(\prod_{i=s}^{k-1} \Phi_{i}\right) \hat{x}_{s}^{-}+\left(\prod_{i=s}^{k-1} \Phi_{i}\right) K_{s}\left(z_{k}^{*}-H_{s} \hat{x}_{s}^{-}\right) \\
& =\hat{x}_{k}^{-}+\left(\prod_{i=s}^{k-1} \Phi_{i}\right) K_{s}\left(z_{k}^{*}-H_{s} \hat{x}_{s}^{-}\right) \\
& =\hat{x}_{k}^{-}+\delta \hat{x}_{k}
\end{aligned}
$$

From the equation above, the correction of state estimate, $\delta \hat{x}_{k}$, is obtained. It is observed that the correction is fulfilled by simply adding $\delta \hat{x}_{k}$ to the propagated state $\hat{x}_{k}^{-}$when the delayed measurement $z_{k}^{*}$ arrives. This relationship may not hold in all filter models. Because the state transition matrix, Eq. (16), is independent of UAV state in this case, however, we do not need to roll back the propagation steps.

In a similar manner, the error covariance matrix is updated when $z_{k}^{*}$ arrives. Although it will lead to suboptimal estimates of the state vector and error covariance matrix in a period of $k$-s samples, the filter state and covariance will once

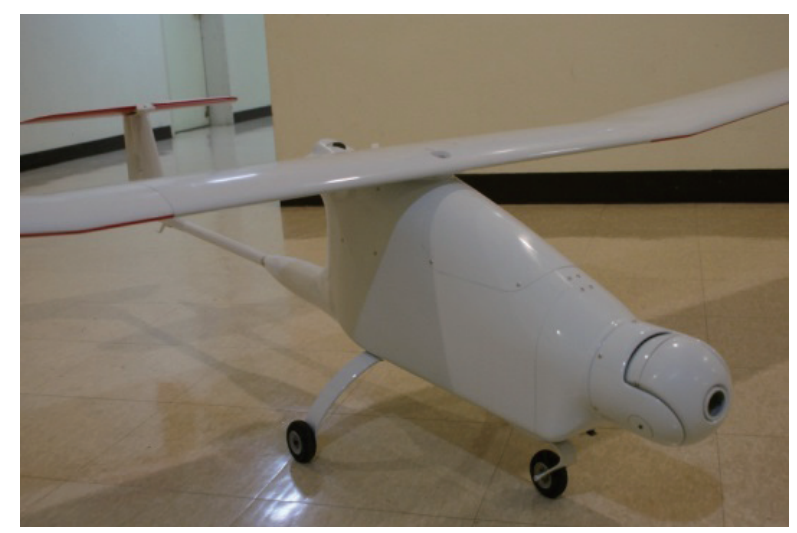

Fig. 7. HeXUS F-3 used for the flight test. It is equipped with a twoaxis gimbal system again be optimal after the correction step.

\section{Experimental Set-up}

\subsection{Flight Test Hardware}

In this section, details of the flight hardware are described. The flight hardware includes the airframe, flight control computer (FCC), onboard vision system (OVS), GPS-aided inertial navigation system (also referred to as INS/GPS), and the camera/gimbal system.

Hexus F-3 (Fig. 7) is based on a Remoeye-006 UAV airframe with the characteristics in Table 1. The FCC consists of the main processor, a servo controller, and two RF modems. With the modems, the UAV communicates both with a ground control system (GCS) and a RC controller. Guidance, navigation, and control algorithms are executed by the main processor. Signals for autonomous flight are then sent to the servo controller. Our UAV is equipped with 3DM-GX3-45, a commercial GPSaided inertial navigation system. It consists of AHRS, GPS, and EKF. It is certain that the accuracy of the INS, provided by 3DM-GX3-45, will directly affect the performance of the vision-aided navigation system. In particular, vision-based solutions are very sensitive to vehicle attitude. Its attitude accuracy is $0.35^{\circ} \mathrm{RMS}$ for roll/pitch and $1.0^{\circ} \mathrm{RMS}$ for heading This experiment will verify that the onboard vision system can successfully calibrate the low-cost INS.

Table 1. Specifications of the HeXUS F-3

\begin{tabular}{ll}
\hline Element & Description \\
\hline Wing Span & $2.59 \mathrm{~m}$ \\
Length & $1.72 \mathrm{~m}$ \\
Power system & Electrically Powered, $720 \mathrm{~W}$ \\
Basic Flying weight & $6.5 \mathrm{~kg}$ \\
Payload including avionics & $0.7 \mathrm{~kg}$ \\
Endurance & $20 \mathrm{~min}$ \\
Gimbal & 2 -axis \\
\hline
\end{tabular}

Table 2. Specifications of the flight control computer (FCC)

\begin{tabular}{ll}
\hline Element & Description \\
\hline Main Processor & TMS320F28335 (Texas Instrument) \\
Servo Controller & AT91SAM7S64 \\
Communication & UART 8 ports (64 bytes FIFO buffer) \\
Memory & NAND Flash Memory \\
\hline
\end{tabular}


The OVS is based on the TMS320DM648 processor, the specification of which is presented in Table 3. It has single core operating at high clock. Output images are transmitted by a $5.8 \mathrm{GHz}$ video transmitter to the mission control system (MCS). The overall avionic system is shown in Fig. 8.

\subsection{Flight Test Algorithms}

The purpose of the flight test was to verify that the proposed vision-aided relative navigation provides the UAV sufficient information for flight control to loiter around a target at the moment of GPS blackout. The guidance law and flight scenario to fulfill the purpose will be described in the following subsections.

\subsubsection{Guidance Law}

For tracking a target continuously, the fixed-wing UAV needs to loiter around the target. In this study, standoff vector field guidance was applied. The standoff vector field is defined as

$$
\left[\begin{array}{c}
\dot{x}_{r} \\
\dot{y}_{r}
\end{array}\right]=\frac{-s_{r}}{\sqrt{r^{4}+\left(p^{2}-2\right) r_{d}^{2} r^{2}+r_{d}^{4}}}\left[\begin{array}{l}
\frac{r^{2}-r_{d}^{2}}{r} x_{r}+p r_{d} y_{r} \\
\frac{r^{2}-r_{d}^{2}}{r}-p r_{d} x_{r}
\end{array}\right]
$$

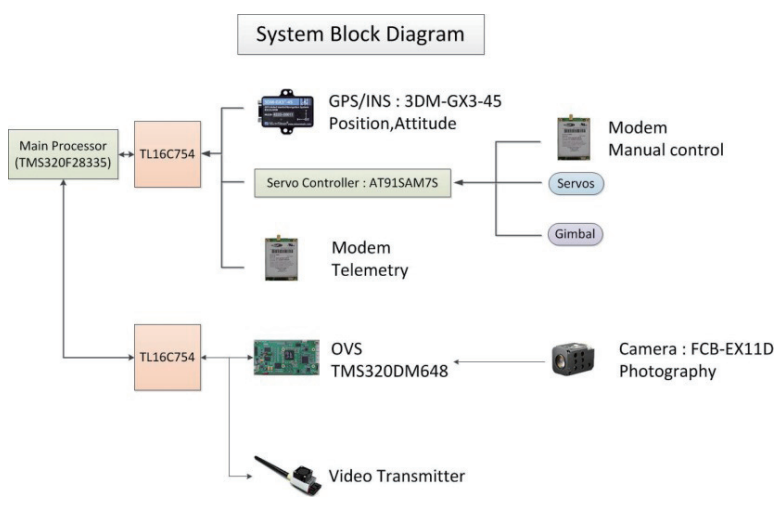

Fig. 8. System block diagram of our UAV.

Table 3. Specifications of the onboard vision system (OVS)

\begin{tabular}{ll}
\hline Element & Description \\
\hline Main Processor & TMS320DM648 (Texas Instrument) \\
Clock & $891 \mathrm{MHz}$ \\
Memory & $512 \mathrm{~KB} \mathrm{L2} \mathrm{Cache,} \mathrm{128MB} \mathrm{DDR2} \mathrm{RAM}$ \\
Flash Memory & $512 \mathrm{MB}$ \\
Video Input / Output & UART 8 ports (64 bytes FIFO buffer) \\
AD Convertor & Input: 4 (NTSC) / Output: 1 (NTSC) \\
Communication & RS-232 1 port \\
Operation System & DSP/BIOS RTOS \\
\hline
\end{tabular}

where $\left[x_{r}, y_{r}\right]^{T}$ is the relative UAV position with respect to the target, $r$ is the distance to the target, $r_{d}$ is the desired standoff distance, $s_{r}$ is the desired relative speed, and $p$ is a non-dimensional parameter that relates radial motion and tangential motion. The ground track angle command is given as the tangential direction of the vector field. Refer to the paper [11] for further details.

The vector field requires only the relative position of the UAV and calculation of Eq. (23) to produce a guidance command. This is appropriate as a real-time solution in that it needs relatively low computational power and little information. We used this guidance law for both a GPS-aided flight and a GPS-denied flight as an outer loop of a typical PID flight controller.

The guidance loop controls ground track angle, speed, and height. The ground track angle command is generated by the vector field guidance for a circular flight around the target. The speed and height are commanded to be constant during the flight.

\subsubsection{Flight Scenario}

At the start, the UAV approaches to the target and loiters around the target using the equipped GPS-aided inertial navigation unit. After the vision system starts to track the target, we let the UAV enter a GPS blackout environment.

The GPS-aided inertial sensor output is logged for later comparison. Because the UAV is not equipped with a barometer, the height information of the GPS data is assumed to be a height measurement with an additional random error, the standard deviation of which is $1 \mathrm{~m}$. The flight conditions and settings are presented in Table 4 .

\section{Flight Test Results}

Using the UAV system described above, in conjunction with the visual relative navigation technique described in

Table 4. Flight conditions and settings

\begin{tabular}{ll}
\hline Parameter & Value \\
\hline Flight Mode & Loitering around the target \\
Flight Altitude & about $140 \mathrm{~m}$ \\
Speed & about $15 \mathrm{~m} / \mathrm{s}$ \\
Desired Standoff Distance & $150 \mathrm{~m}$ \\
Guidance/Control frequency & $50 \mathrm{~Hz}$ \\
Navigation Frequency (GPS/INS) & $50 \mathrm{~Hz}$ \\
Navigation Frequency (Vision/INS) & $25 \mathrm{~Hz}$ \\
- State Propagation & $25 \mathrm{~Hz}$ \\
- Measurement Update & $5 \mathrm{~Hz}$
\end{tabular}


this paper, we conducted a flight test. The flight trajectory is shown in Fig. 9. For comparison, the trajectory from INS/ GPS is drawn along with the result of vision-aided navigation system, denoted as INS/vision in the figure. The UAV was able to loiter around the target and let the vision system track the target continuously. The vision-aided navigation system provided the UAV with position information successfully to keep the UAV stable in the GPS-denied environment.

Figure 10 shows errors of visual estimates in the three axes. In this figure, trajectory from the commercial GPSaided inertial navigation system mounted on the UAV is regarded as 'true.' Because the INS/GPS sensor system uses a single GPS, in fact, its output cannot be considered as a 'real' trajectory. The INS/GPS sensor used in this study has a position accuracy of 2.5 m RMS horizontal and 5.0 m RMS vertical. For investigating the accuracy of the vision-aided navigation system, a differential GPS (DGPS) system would be helpful to improve the accuracy.

It can be seen that the estimation errors in the East and

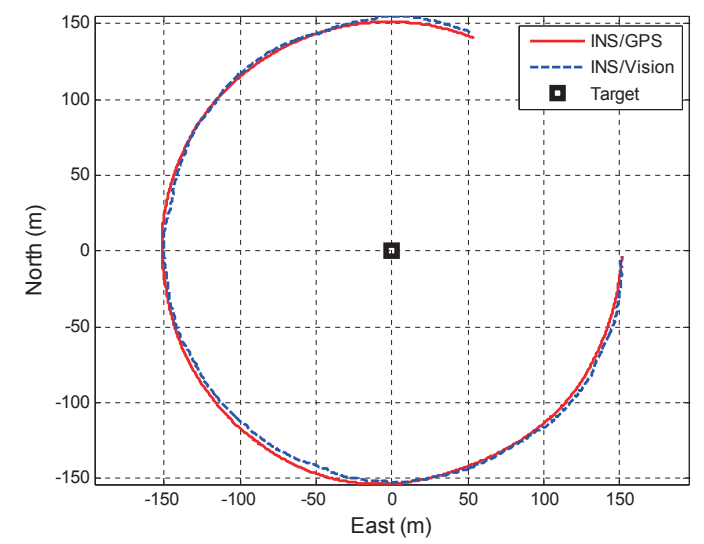

Fig. 9. Flight trajectory of the UAV. The UAV is loitering around the target in a counterclockwise direction. Only part of the trajectory is drawn for simplicity.
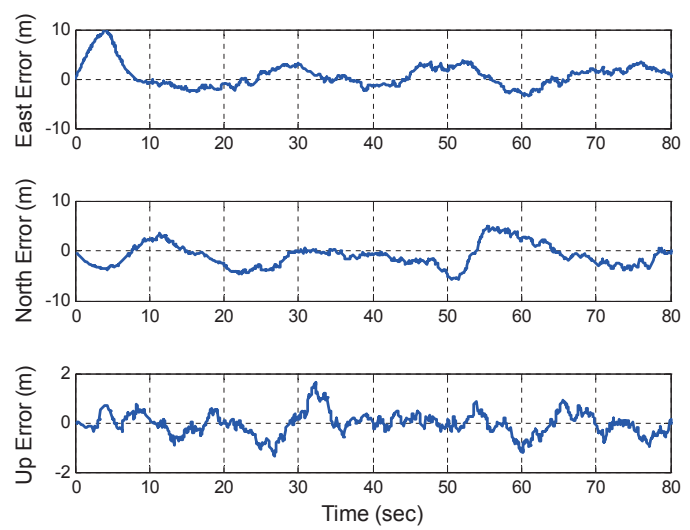

Fig. 10. Time history of estimation errors. In this case, INS/GPS output is assumed to be 'true.'
North directions are in the form of sinusoidal functions in the opposite phase. A small bias in the gimbal angle measurement can lead to such results. The attitude error of the INS is also directly related to the navigation results. One of the measures of performance for the navigation system is to compare the actual distance to the desired distance defined in the vector field guidance because the accuracy of the navigation system has an effect on the guidance performance. The time history of distance to the target is shown in Fig. 11. It shows that the UAV maintained a distance larger than the 'desired' distance during flight. However, the error was less than $5 \mathrm{~m}$, which is satisfactory in that the UAV still managed to loiter around and visually track the target.

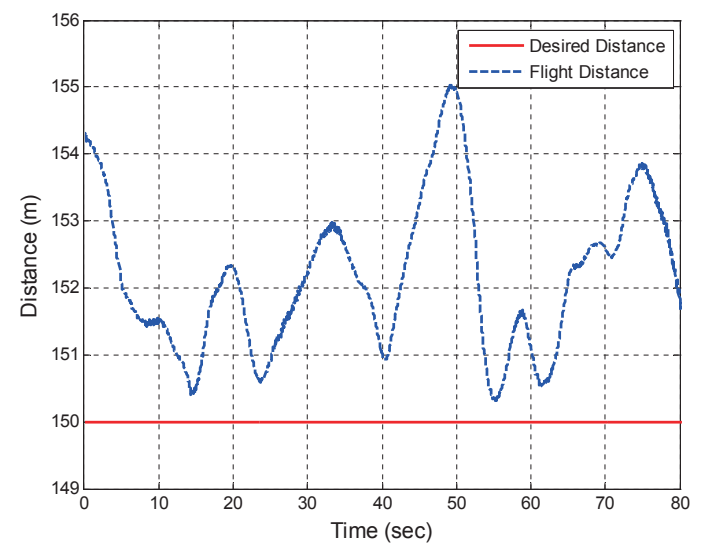

Fig. 11. Time history of distance to the target.

\section{Conclusions}

In this paper, we introduces a system for visual target tracking and the relative navigation of a fixed-wing unmanned aerial vehicle. We placed emphasis on techniques and algorithms for implementing practical real-time systems. The image processing algorithms and navigation filter design are discussed in detail. The primary contribution of the paper is that we showed, by flight tests, that the visual relative navigation enabled position-keeping without GPS data. The flight test was conducted with a fixed-wing UAV with a two-axis gimbal system. The guidance error was less than $5 \mathrm{~m}$ and the target was tracked stably by the vision system during flight. The system is expected to be exploited in a fail-safe mode in a GPS blackout and target surveillance during a war. Any visible and unknown landmark or terrain feature can be used as a target. Future research will include expanding the techniques to more elaborate missions in GPS-denied environments. 


\section{Acknowledgements}

This research was conducted with governmental funds from the Technological Innovation Project of the Ministry of Knowledge Economy (MKE). The project code is 10041880.

\section{References}

[1] King, A. D., "Inertial navigation-past, present, and future", In IEE Colloquium on Airborne Navigation Systems Workshop. Digest 1997/169, 1999.

[2] Johannessen, R., "The role of GPS in flight calibration", In IEE Colloquium on Current Future Trends Flight Calibration Radio Navigational Aids, 1991.

[3] Djederich, P., "Global navigation satellite systems", In IEE Airborne Navigational Aids, 1998.

[4] JAVNTS Center., "Vulnerability assessment of the transport infrastructure relying on the global positioning system", Office of the Assistant Secretary for Transportation Policy. U. S. Department of Transportation, 2001.

[5] Watanabe, Y., Fabiani, P. and Vesnerais, G. L., "Simultaneous visual target tracking and navigation in a
GPS-denied environment", IEEE International Conference on Advance Robotics, Munich, 2009.

[6] Comaniciu, D., Ramesh, V. and Meer, P., "Kernel-based object tracking", IEEE Transaction on pattern analysis and machine intelligence, Vol. 25, No. 5, 2003, pp.564-577.

[7] Barber, D. B., Redding, J. D., McLain, T. W., Beard, R. W. and Taylor, C. N., "Vision-based target geo-location using fixed-wing miniature air vehicle", J Intell Robot Syst, Vol. 47, No. 4. 2006, pp. 361-382. DOI:10.1007/s10846-006-9088-7

[8] Brown, R. and Hwang, P., Introduction to Random Signals and Applied Kalman Filtering, John Wiley \& Sons, 1997

[9] Bletzacker, et al., "Kalman Filter Design for Integration of Phase III GPS with an Inertial Navigation System" Computing Applications Software Technology Technical Papers, Los Alamitos, CA, 1988.

[10] Larsen, T. D., Andersen, N. A. and Ravn, O., "Incorporation of time delayed measurements in a discretetime Kalman filter" Proceedings of the 37th IEEE Conference on Decision \& Control, Tampa, Florida, 1998.

[11] Lim, S., Kim, Y., Lee, D. and Bang, H., "Standoff target tracking using a vector field for multiple unmanned aircrafts", Journal of Intelligent \& Robotic Systems, Vol. 69, No. 1-4, pp. 347-360. 2013. DOI: 10.1007/s10846-012-9765-7 